\title{
Principles for national and regional guidelines on cardiovascular disease prevention
}

\author{
Sidney Smith, Janet Voûte and Valentin Fuster
}

Cardiovascular disease (CVD) is the leading cause of global mortality resulting in nearly 17.5 million deaths worldwide in 2005. Whereas the causes of this epidemic involve the same risk factors, the approaches to its control and prevention can differ in each geographic region because of cultural, social, medical and economic circumstances. In recognition of the need for effective implementation of guidelines to curb the growing worldwide mortality from CVD, the World Heart Federation (WHF) has set forth principles upon which every nation can develop a policy on CVD prevention. The recommended strategies involve treatment of high-risk patients using medical therapies whose benefits have been confirmed by large randomized clinical trials, and the parallel institution of behavioral modification for the general population focused on diet, physical activity and prevention of smoking.

As $80 \%$ of CVD-related mortality now occurs in countries with developing economies, it is imperative that governments work in partnership with national societies of health-care providers and foundations to develop guidelines that reflect national or regional priorities and resources. The greatest cost-effectiveness for prevention of CVD generally occurs through the treatment of patients at high short-term risk, whether that risk is based on a previous CVD event or determined by high global risk. Among populations at lower short-term risk of CVD, programs directed toward preventing use of tobacco products and encouraging physical activity and proper diet are public health measures that can be highly cost-effective. The health-care budget for each nation should reflect a thoughtful determination, which weighs the specific benefits for primary and secondary prevention programs based on local epidemiologic factors. Importantly, every effort should be made to address financial constraints placed upon the health-care system.

The WHF recommends 10 strategic principles for the development of national clinical "....health-care

providers,

national

societies and

foundations,

and

governments

must act now

in partnership

to implement

guideline-

based

programs and

legislation..."

$S$ Smith is Chairman of the World Heart

Federation Scientific

Advisory Board and

Professor of Medicine at the University of

North Carolina at

Chapel Hill. J Voute

is Chief Executive

Officer of the World

Heart Federation and

$V$ Fuster is the Editor-

in-Chief of Nature

Clinical Practice

Cardiovascular

Medicine.

\section{Competing interests}

The authors declared they

have no competing interests

www.nature.com/clinicalpractice doi:10.1038/ncpcardio0655 guidelines: (i) governments, national societies and foundations should collaborate to develop clinical and public health guidelines for CVD prevention that target risk factors; (ii) evidence-based guidelines should incorporate professional judgment on the translation of such evidence into effective and efficient care, addressing all areas of CVD risk; (iii) the assessment of total CVD risk should be based on epidemiological risk factor data appropriate to the population to which it is applied; (iv) policy recommendations and guidelines should emphasize the importance of a total risk reduction for CVD prevention; (v) the intensity of interventions should be a function of the total CVD risk, with lower treatment thresholds for higher-risk patients than for low-risk patients; (vi) national cardiovascular societies and foundations should promote routine, prospective collection of validated national vital statistics on the causes of CVD and outcomes of patients with CVD for use in the development of national policies; (vii) national professional societies should inform policy makers of risk factor targets and drug therapies for CVD prevention that are culturally and financially appropriate to their nation, and ask their governments to incorporate CVD prevention into legislation whenever relevant; (viii) national professional societies and foundations should facilitate CVD prevention through education and training programs for health-care professionals; (ix) national professional societies should assess the achievement of lifestyle, risk factor and therapeutic targets defined in the national guidelines; $(x)$ health-care professionals should include CVD prevention as an integral part of their daily clinical practice.

If we are to succeed in our efforts to curb the increasing burden of CVD worldwide, health-care providers, national societies and foundations, and governments must act now in partnership to implement guideline-based programs and legislation directed toward the prevention of CVD. 